Bull. Mater. Sci., Vol. 4, No. 2, April 1982, pj), 85-102. (C) Printed in India.

\title{
Capillary equilibrium and stability in liquids under microgravity
}

\author{
J M HAYNES \\ Department of Chemistry, University of Bristol, Bristol, UK \\ MS received 17 June 1980
}

\begin{abstract}
The conditions governing capillary equilibrium and stability of a fluid interface are stated. A distinction is drawn between hydrostatic (or Laplace) and diffusional (or Kelvin) equilibria, which is often of practical importance in the behaviour of real liquids. In the light of these principles, the fluid physics experiments proposed for the first Spacelab payload are discussed, with particular reference to the rotational stability of a fluid cylinder in microgravity and to processes used to grow large single crystals of pure materials.
\end{abstract}

Keywords. Capillary equilibria; stability; capillarity; hydrostatic equilibria ; diffusional equilibria.

\section{Surface tension and capillarity}

\subsection{The nature of surfaces}

In the absence of external fields, bulk phases of matter at rest are characterised by the isotropic and uniform nature of such intensive properties as density, pressure, temperature and chemical potential. At boundaries between phases, on the other hand, there are macroscopic discontinuities of structure and chemical constitution; but this macroscopic view does not accurately reflect the state of the system at the molecular level.

In the interior of a bulk fluid phase the intermolecular force field, suitably timeaveraged, is uniform and isotropic. At the molecular scale, the word "interior" implies that the region under consideration is not within intermolecular interaction range of another phase. Thus, "boundaries" between phases are of thickness comparable with the normal range of action of intermalecular forces-say a few tens of nanometres. (Close to critical states, where co-operative effects become dominant, boundary thicknesses may be greatly increased.) Although the interface between phases may appear macroscopically sharp, at the molecular level it must involve a cantinuous gradation of properties.

Although the formulation of a satisfactory statistical-mechanical description of an equilibrium fluid interface involves considerable difficulties, an adequate thermodynamic description can be based on a greatly simplified model (Gibbs 1875-78). In the Gibbs model, the two bulk phases adjoining an interface are supposed to continue uniformly in all properties right up to a hy pothetical dividing surface, of strictly zero thickness. Any differences between the content of matter, energy and 
entropy of the real system and the corresponding contents of the two uniform bulk phases of the model are then ascribed, as surface excess properties, to the dividing surface itself.

It will $b \approx$ apparent that the absolute magnitudes of the surface excess quantities derived from the Gibbs model will be highly sensitive to the position chosen for the hypothetical dividing surface. This is so because a small shift of that surface to one side or the other will, in the model, exchange a certain volume of one bulk phase for the same volume of the other, in which the concentrations of matter, energy and entropy will generally be different. The corresponding surface excesses, representing the extent to which the real system differs from two bulk phases separated by a step-wise interface, are therefore sensitive to the way in which the total volume is divided between the two bulk phases, and thus depend critically on the location of the discontinuous phase-junction in the model. Approximate calculations (Defay et al 1966) indicate that Gibbs model surface excess quantities may even change sign when the dividing surface is shifted by a nanometre or two.

By convention, this problem is overcome in the Gibbs model by selecting a particular dividing surface that will render the molar surface excess of one chemical component (usually the major component) precisely equal to zero. The extreme sensitivity of the model now has the result that the dividing surface is located very precisely indeed by this convention. The corresponding relative surface excesses are now defined unambiguously, and provide a firm basis for the development of a complete thermodynamic description of surfaces at equilibrium (Gibbs 1875-78; Defay et al 1966).

It should be emphasised that despite the severely arbitrary nature of the Gibbs model, real interfaces evidently behave physically as though they were the seat of local variations of concentration of matter, energy and entropy. For example, the surface tension force that characterises real interfaces is a manifestation of the existence of a surface excess of energy (althou gh the two are not generally identical), and the presence of surface excesses of so-called surface-active materials, such as soaps, is familiar in the phenomenon of adsorption.

Thus, even though the detailed molecular configuration within an interfacial region at equilibrium (i.e., that which minimises the free energy of the system) remains unknown, the Gibbs model provides a self-consistent alternative which is adequate for many purposes. The properties of interfaces not at equilibrium, on the other hand, usually cannot be discussed in terms of the Gibbs model: this is because their evalution towards equilibrium largely involves the redistribution of matter, etc., within precisely that unknown in homogeneous region of the interface that the Gibbs model seeks to circumvent. For the same reason, transport processes across interfaces fall outside the competence of the Gibbs model.

An alternative model of the interface (Guggenheim 1957) treats it as a third, homogeneous phase of small but finite thickness. Although at first sight this may appear to be one step nearer to reality than the Gibbs model, in fact it suffers from the same drawbacks as the latter, including an inability to describe non-equilibrium states, and in addition requires that two dividing surfaces, rather than one, shoula be defined. More elaborate multilayer models (e.g., Ono and Kondo 1960), whioh tend ultimately towards the full statistical-mechanical solution of the problem, involve increasingly severe difficulties (Defay et al 1966), 
The foregoing remarks have been concerned mainly with fluid interfaces (liquid/ liquid and liquid/gas). Solid surfaces are likewise the seat of surface excesses of energy, etc., althaugh the physical manifestation of the mechanical consequences of the existence of surface tension in solids is hindered by the ability of a solid to exist indefinitely in a non-uniform state of strain. Thus, solid surfaces may display a marked reluctance to attain states of capillary equilibrium, even though certain capillary phenomena involving solids, such as Ostwald ripening, are wellknown.

\subsection{The phenomena of capillarity}

The existence of an excess of free energy associated with an interface means that wark must be expended in any extension of that interface. Thus, the thermodynamic definition of the surface tension $\sigma$ is

$$
(\partial F / \partial A)_{T, V}=\sigma \text {, }
$$

where $F$ is the free energy of the whole system, $A$ the area of the interface, and $T$ and $V$ the temperature and volume of the system. It may be observed that if the interface is extended reversibly, it will retain the same structure as that of the original interface. In a non-equilibrium extension, however, this may not be so: it might, for example, retain the same number of molecules as the original surface, only later relaxing to the equilibrium state as fresh molecules enter the surface to restore the original configuration over the enlarged area of interface. In this case, the surface tension thus defined is called the pure dynamic surface tension. Solids obviously possess the capacity for such non-equilibrium behaviour, but it is also observed that fluid interfacial tensions may depend on the rate of strain, especially if a slowly-diffusing surface-active solute is present.

Operationally, the surface tension is defined as the force normal to a line of unit length in the plane of the surface. Althou gh the tension in a fluid interface may be obtained experimentally by a direct measurement of that force, most methods depend instead on observations, direct or indirect, of the pressure difference across a curved interface (see $\$ 2.1$ ).

For pure liquids, the surface tension is a function of temperature only, (the pressure coefficient being negligibly small except close to the critical state); the quantity $d \sigma / d T$, which represents minus the surface entropy, is generally negative and approximately constant. Thus, $d^{2} \sigma / d T^{2}$, the surface heat capacity, is generally close to zero. The variation of surface tension with temperature for many simple non-associated liquids is of the form

$$
a v^{2 / 3}=K_{E}\left(T-T_{\mathrm{c}}\right)
$$

where $v$ is the molar volume, $T_{c}$ the critical temperature, and $K_{E}$, the Eötvös constant, is about $2 \cdot 1 \times 10^{-7} \mathrm{JK}^{-1}$.

In a solution or liquid mixture, it is cften found that the surface is enriched in the component of lower surface tension: this is the phenomenon of adsorption. Aqueous solutions of ionised salts may sometimes show slight negative adsorption: the surface is depleted in the solute, and the surface tension is a little higher than that of the solvent. For example, the surface tension of 0.1 molar potassium 
chloride solution is $0.16 \mathrm{mN} \mathrm{m}^{-1}$ higher than that of pure water. More usually, however, adsorption of a solute at the surface of a dilute solution is positive and the surface tension is reduced.

Some substances are very strongly adsorbed at fluid interfaces. In the case of aqueaus solutions, such surfactant molecules generally have a polar or hydrophilic head group, attached to a hydrophobic organic chain. Although their solubility may often be appreoiable, such molecules shaw a strong tendenoy to concentrate at the surface with their hydrocarbon 'tails' oriented away from the aqueous phase. At sufficiently high concentrations in solution, the molecules may form aggregates or micelles with their polar groups pointing outwards, thus enhancing their solubility in the bulk phase. Other surfactants may be practically insoluble. and form well-defined monomolecular layers on the surface. Surfactants have the property that even in very low concentrations they may reduce the surface tension of water by a factor of three or more. This fact emphasises the importance of cleanliness in surface chemistry: a relatively small number of foreign molecules may alter entirely the nature of a surface.

Because of the excess of free energy associated with a surface, there is a tendency for fluid interfaces to adopt configurations that minimise their area (in the absence of external fields), subject to whatever boundary conditions may be imposed. This will be treated more fully in the discussion of capillary equilibrium in $\S 2$.

\section{Capillary equilibrium and stability}

\subsection{Basic equations}

When a membrane, subject to a tension, is curved, a necessary oondition for its mechanical equilibrium is that there should be a hydrostatic pressure difference between the media on its two sides. This general condition applies equally to fluid interfaces subject to a surface tension. By equating the pressure-volume work done in an infinitesimal departu re from equilibrium to the product of surface tension and change in area, and applying an appropriate geometrical condition, the hydrostatic pressure difference $\triangle P$ is found to be given by the Laplace equation:

$$
\triangle P=\sigma C
$$

where $\sigma$ is the surface tension.

The mean curvature $C$ is defined as

$$
C=1 / r_{1}+1 / r_{2}
$$

where $r_{1}$ and $r_{2}$ are the principal radii of curvature. Expressing the curvature in terms of differential coefficients of the co-ordinate $z$ with respect to orthogonal co-ordinates $x$ and $y$,

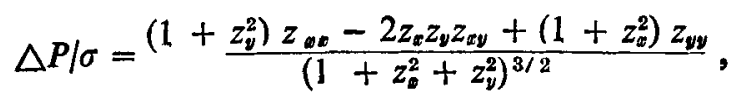

where $z_{y}=(\partial z \mid \partial y)_{z}$, etc. 
In the case of a liquid/vapour interface, a principal curvature is conventionally given a positive sign when its centre of curvature lies in the liquid phase. No consistent convention exists in the case of liquid/liquid interfaces, though in the discussion of axisymmetric interfaces it is convenient to regard a curvature as positive when its centre lies toward the inner side of the interface. A surface is said to be synclastic or anticlastic according as its princif al curvatures are of like or unlike sign.

Example: A spherical droplet of liquid has a synclastic surface of positive curvature; the two principal radii are identical, being equal to the radius of the sphere, $r$, and, according to the Laplace equation, there is an excess pressure inside the droplet given by

$$
\triangle P=2 \sigma / r \text {. }
$$

The excess hydrastatic pressure inside such a drop will increase the chemical potential of the liquid therein and this will be manifested by an increase in its equilibrium vapour pressure. The relationship between curvature and vapour pressure is given by the Kelvin equation:

$$
\frac{R T}{v_{l}} \ln \left(p / p^{0}\right)=\sigma C,
$$

where $R$ is the gas constant, $v_{b}$ the molar volume of the liquid, $p^{0}$ its normal saturation vapour pressure (above a plane interface) at the temperature $T$, and $p$ the equilibrium vapour pressure above the interface of mean curvature $C$. Thus, $p$ is greater or less than $p^{0}$ according as $C$ is positive or negative.

Example: A liquid is confined inside a cylindrical capillary tube which it wets, so as to form a short thread with hemispherical ends. The liquid/vapour interfaces are negatively curved (centre of curvature in the vapour phase), and hence the liquid exerts a reduced vapour pressure.

Table 1 gives typical values of $\triangle P$, the hydrostatic pressure difference according to the Laplace equation, and $p / p^{0}$, the relative vapour pressure according to the Kelvin equation, for spherical water drops and water varour bubbles $(\sigma=$ $72 \mathrm{mN} \mathrm{m}^{-1}$ ) of various radii, $r$, at $20^{\circ} \mathrm{C}$.

Table 1。

\begin{tabular}{cccc}
\hline$r / m$ & $P /$ bar & $p / p^{\circ}$ (bubble) & $p / p^{\circ}$ (droplet) \\
\hline $1 \times 10^{-5}$ & 0.144 & 1.0001 & 0.9999 \\
$1 \times 10^{-6}$ & 1.44 & 1.0011 & 0.9989 \\
$1 \times 10^{-7}$ & 14.4 & 1.0107 & 0.9894 \\
$1 \times 10^{-8}$ & 144 & 1.1122 & 0.8991 \\
$5 \times 10^{-9}$ & 288 & 1.2370 & 0.8084 \\
$2 \times 10^{-9}$ & 720 & 1.7017 & 0.5876 \\
$1 \times 10^{-9}$ & 1440 & 2.8959 & 0.3453 \\
\hline
\end{tabular}


In calculating these figures, it is assumed that the liquid is incompressible, and that it has its bulk value of surface tension. The first of these rcstrictions is easily relaxed; it turns out that for normal liquids not near the critical state the compressibility correction is practically negligible for radii greater than about $10 \mathrm{~nm}$. It is to be expected that droplets of this size and smaller will contain too few molecules to be treated thermodynamically as a bulk phase and that in this region the surface tension may depart substantially from its bulk value.

Returning to equation (3), we note that within a stationary fiuid not subject to external fields the hydrostatic pressure must be uniform. Since, for mechanical equilibrium in a fluid interface, the tension must also be uniform, the Laplace equation indicates that the mean curvature $C$ must also. be uniform over the whole interface. The study of uniform-curvature (or homoclastic) surfaces is an important part of microgravitational fluid physics (see $\$ 2.3$ ), although it has been of interest to mathematicians for at least 150 years (see Maxwell 1876, and earlier references cited therein).

When a fluid interface meets a plane solid surface of uniform composition, it may either do so asymptotically- the case of spreading-or it may form an intersection at a finite angle $\theta$, the so-called contact angle, measured throu gh the liquid phase. If the contact angle is less than $90^{\circ}$, the liquid is said to wet the solid. The contact angle is a measure of the relative strengths of cohesion within the liquid and adhesion between the liquid and the solid. It can be shown (Johnson 1959 ; Everett and Haynes 1972a) that the free energy is minimised when Young's equation is obeyed:

$$
\sigma_{s v}-\sigma_{b l}=\sigma_{l v} \cos \theta
$$

where $s, l$ and $v$ stand for solid, liquid and vapour, and $\sigma$ denotes an interfacial tension between the phases indicated by the subscripts.

If the solid surface has a discontinuity of slope at a line, e.g., an edge, or has a boundary between macroscopic areas of different wettability, the contact angle may become indeterminate, within limits, at that line. In such cases, a moving contact line may be halted at the discontinuity while the contact angle swings between its limiting values; while the contact line is in such a position, the configuration of the rest of the interface may change without violating the boundary condition. This is the phenomenon of canthotaxis, or 'anchoring at an edge' which will be made use of in the fluid physics module experiments (q.v.) and which is also of importance in the wetting of porous media. The mirimum scale of heterogeneity that will give rise to canthotaxis is determined by the ability of the fluid interface to find homoclastic configurations conforming to discontinuous boundary conditions. At the molecular scale, the surface may be able to adopt other configurations, not satisfying equation (5), within the non-uniform force field of the solid. Nevertheless, hysteresis of the contact angle is often marked on apparently homogeneous surfaces.

The minimisation of the fluid interfacial area that is necessary for equilibrium, in the absence of external fields, is modified when a solid is in contact with the fluids, since three kinds of interface are now involved. We require instead, for equilibrium,

$$
0=\sigma_{\varepsilon l} d A_{\varepsilon l}+\sigma_{s v} d A_{s n}+\sigma_{l v} d A_{l v}
$$


where each $A$ represents an interfacial area. Using equation (8), together with the fact that the area of the solid is constant, we find:

$$
0=\sigma_{l v} d\left(A_{l v}-A_{s l} \cos \theta\right) \text {. }
$$

Defining $A_{l v}-A_{s l} \cos 0$ as the effective area, $A^{\text {eff }}$, we thus find that equilibrium corresponds to extremisation of the effective area.

The effectiva area also obeys a geometrical relationship known as the Gauss equation:

$$
d A^{\mathrm{eff}} / d V=C,
$$

where $V$ is the volume of the phase enclosed by the interface whose mean curvature is $C$ (Everett and Haynes 1972b).

In practice, it may often be possible to achieve states of partial equilibrium, because of differences in the rates of hydrodynamic and difiusional flow. Thus, a liquid having both low viscosity and low vapour pressure may rapidly attain an equilibrium shape, in accordance with equation (5), but may be extrcmely slow to adopt its equilibrium vapour pressure according to equation (7). Moreover, it can be seen from table 1 that the driving force for the two kinds of equilibrium may be of very different magnitu des for surfaces of not too great a curvature. It is therefore useful to distinguish between Laplace (or hydrostatic) equilibrium and Kelvin (or diffusional) equilibrium (Everett and Haynes 1972b). The fluid physics madule experiments described in $\$ 3$ below are concerned solcly with Laplace equilibrium.

The distinction is emphasised when we consider the stability of capillary equilibrium, i.e., whether a given stationary value of free energy is a minimum (stable or metastable), a maximum (vnstable) or a horizontal inflection (neutral). When only capillary forces are considered, the conditions for stability are (Hayncs 1977)

$$
\begin{array}{ll}
\left(\partial^{g} A^{\text {eff }} / \partial \xi^{2}\right)_{V}>0 & \text { (Laplace), } \\
\left(\partial^{2} A^{\text {eff }} / \partial V^{2}\right)_{\xi=0}>0 & \text { (Kelvin), }
\end{array}
$$

where $\xi$ is a parameter describing departure from uniform curvature. In conjunction with the Gauss equation, the second condition becomes

$$
(\partial C / \partial V)_{\xi=0}>0 \quad \text { (Kelvin). }
$$

Many equilibrium canfigurations may be stable in one sense and unstable in the other.

Example: The spherical liquid droplet referred to earlier is obviously a stable Laplace equilibrium shape, since any deformation from sphericity will increase its interfacial area. If, however, it is also in Kelvin equilibrium, the surrounding vapour will be slightly supersaturated [compare equation (7) and table 1]. If now it suffers a small pasitive fluctuation of volume, the requircd supersaturation will be reduced and so the drop will continue to grow indefinitely by condensation. Similarly, if it momentarily decreases from its original size, it will now exert an increased vapour pressure and thus will continue to shrink until it has all evaporated. Since the drop has a positive curvature of $2 / r$ and a volume of $4 \pi r^{3} / 3$, we see that $d C / d V=-1 / 2 \pi r^{4}<0$, and it does not meet the Kelvin stability condition of equation (12c). A spherical drop is therefore Laplace-stable and Kelvin-unstable. 
In the next section, more general conditions for capillary equilibrium will be stated and particularised to a few cases of specific interest.

\subsection{General conditions for capillary equilibria}

In this section consideration will mainly be restricted to constant-volume configurations of an incompressible and involatile liquid. The latter restriction means that, unless otherwise stated, the surrounding, second fluid phase can be regarded as inert, and Kelvin equilibrium can be ignored.

Consider a fixed mass of an inert, invalatile liquid, at constant and uniform temperature, in contact with an inert and rigid solid forming a uniform contact angle. The system is subject to a steady gravitational field in the negative $z$-direction and is in steady rotation with angular velocity $\omega$ about a fixed axis $X Y$ (figure 1).

The energy of the system at constant temperature will depend on the pressure and volume of the liquid and, in addition, will contain terms arising from surface energy, gravitational potential energy and rotational kinetic energy. (Adaditional canservative force fields, such as electrical, magnetic, and intermolecular repulsive and attractive fields, could be introduced, if desired).

Equilibrium states of the system will correspond to stationary values of the energy with respect to permitted variations, subject to the boundary constraints of constant volume and contact angle. At energy minima the equilibria will be stable (or metastable), and at the vanishing point of an energy minimum the system will reach a stability limit, which may lead to an abrupt and thermodynamically irreversible change of configuration. Thus, at equilibrium [ $c f$. equation (18) of Everett and Haynes (1972a)]:

$$
\begin{aligned}
d F & =0 \\
& =-\int p \delta V+\sigma d A^{\mathrm{eff}}-g \rho z^{*} d V-g \rho V d z^{*}+\rho \omega^{2} V k d k+\frac{1}{2} \rho \omega^{2} k^{2} d V
\end{aligned}
$$



Figure 1. Capillary equilibrium of a rotating fluid mass adhering to a solid, in a gravitational field. 
where $p$ is the capillary pressure difference in a particu lar volume clement of liquid $\delta V, \rho$ is the density of the liquid (that of the surrounding medium being taken as zero), $z^{*}$ is the $z$-coordinate of the centre of mass of the liquid relative to the level at which $p=0$, and $k$ is the radius of gyration of the liquid mess. The surface energy terms, including the contact angle, arc collectcd in the tcrm in effective area. $A^{\text {ert }}$ [see equation (10)].

The $-p d V$ term in equation (13) appears as an integral over the whole system because $p$ is a function of position within a gravity-affectc and/or rotating liquid drop. The Laplace equation, relating capillary pressure to interfacial curvature will nevertheless be locally valid everywhere. Thus, we may write

$$
\mathbf{0}=\sigma\left(-\int C \delta V+d A^{\mathrm{eff}}\right)-g \rho\left(z^{*} d V+V d z^{*}\right)+\rho \omega^{2} k\left(V d k+\frac{1}{2} k d V\right) .
$$

If we set $g=\omega=0, C$ is position-independent and we rccover the Gauss equation [equation (11)]. Alternatively, if we accept the guneral validity of the Gauss equation, which is bascd on geometry rathir than capillarity, we have demonstrated that Young's equation [equation (8)], which is assumcd in the definition of $A^{\text {eff }}$, is indeed a condition of thermodynamic cquilibrium.

The equilibrium condition can be made dimensionless by introducing the lengths

$$
\begin{aligned}
& \left.a^{2}=\sigma / \rho g \quad \text { (i.c., } \mathrm{B} \mathrm{O}=a^{2} / R^{2}\right), \\
& \left.b^{3}=\sigma / \rho \omega^{2} \quad \text { (i.e., } \mathrm{B}{ }^{\prime}=b^{3} / R^{3}\right) .
\end{aligned}
$$

In the context of microgravititional behaviour, where a bccomes infinite, we nced write merely

$$
\frac{k}{b^{3}}\left(V d k+\frac{1}{2} k d V\right)-\int C \delta V+d A^{\text {eff }}=0 .
$$

This equation describes the equilibrium of a rotating fluid mass in negligible gravity. Its consequences will be revisited in $\S 3 \cdot 2$.

An illustration of the use of equation (17) is provided by the so-called spinning drop method for determining the interfacial tension between two fluids. The denser fluid, containing a bubble of the less dense fluid, is confined within a long capillary tube which rotates rapidly about its axis. At sufficiently high rotation rates the bubble may be considered as a cylinder of axial ratio (length)/(radius) $=\lambda$. The curvature of the bubblc is uniform and its volume is constant; hence

$$
\begin{aligned}
& \int C \delta V=0, \\
& d V=\pi r^{3} \lambda+3 \pi r^{2} \lambda d r=0,
\end{aligned}
$$

and $\quad d A^{\text {eff }}=-2 \pi r \lambda d r$.

Since, for a cylinder

$$
k=r / \sqrt{2}
$$

it follows that

$$
\sigma=\rho \omega^{2} r^{3} / 4 \text { or } \mathbf{B}_{0_{R}}=1 / 4 \text {. }
$$


This relationship, due to Vonnegut (1942), is widely used for the determination of interfacial tension, especially in thase systems, of interest to the petroleum industry, where $\sigma$ is extremely low (Cayias et al 1975).

\subsection{Axisymmetric homoclastic surfaces}

When both $a$ and $b$ become infinite, equation (13) describes surfaces for which $p$ is not a function of position and whose mean curvature is therefore uniform, i.e., homoclastic surfaces. The beheviour of soap films demonstrates that an infinite variety of such surfaces can be formed to satisfy a wide variety of boundary conditions, but, althou gh they are so readily realisable in practice, only a limited set of such surfaces is capable of analytical description. These are the surfaces having an axis of symmetry, for which $z_{y}=z_{x}$. Under these circumstances equation (5) can be integrated yielding solutions in terms of elliptic and/or trigonometric functions.

There are only five classes of axisymmetric surfaces of uniform mean curvature. They are conveniently visualiscd through their relationship with the five classes of conic section (Maxwell 1876).

If a conic section (circle, ellipse, straight line, parabola or hyperbole) is rollcd, without slipping, along a straight line, its focus will trace out a particular locus. If that locus is now rotated about the rolling path, it will farm one of the five classes of axisymmetric homoclastic surface (cylinder, unduloid, sphere, catenoid and nadoid). These configurations are thus surfaces of revolution of conic focal roulette curves.

The rolling of a circle is easily visualised: its centre traces out a straight line parallel to the rolling path, which on rotation about that path yields a cylinder.

Each fosus of a rolling ellipse traces out a periodically wavy curve; on rotation this yields the unduloid, whose amplitude increases as the eccentricity of the generating ellipse becomes greater.

The "rolling" of a straight line is represented by an end-over-end somersaulting motion. The foci of the line are at its ends, and each generates a series of semicircles, which form spheres an revolution about the rolling path.

The focus of a rolling parabola traces out a catenary-the curve of a heavy chain suspended by its ends-and on revolution about the rolling path this generates the catenoid.

Finally, we consider the rolling of a pair af hyperbolae. Beginning with the hyperbolae orientated sa that the $x$-axis (the rolling path) coincides with one of their common tangents (figure 2), the points of contact of the upper and lower branches are at minus and plus infinity. Rolling in a clockwise direction and keeping the upper branch in contact with the $x$-axis, its contact point moves from minus to plus infinity, whereupon the lawer branch contacts the $x$-axis at minus infinity. Meanwhile, the upper and lower faci trace the paths 123 and $1^{\prime} 2^{\prime} 3^{\prime}$ respectively (figure 2). Rolling the hyperbolae anti-clockwise and keeping the lower branch in contact, the point of contact again moves from minus to plus infinity, and the focal roulettes follow the paths 3.45 and $3^{\prime} 4^{\prime} 5^{\prime}$. Rotation of these loci about the rolling path generates the nodoid surface. We notice the ourious fact that the focal roulette curves, whioh are obviously complementary, 


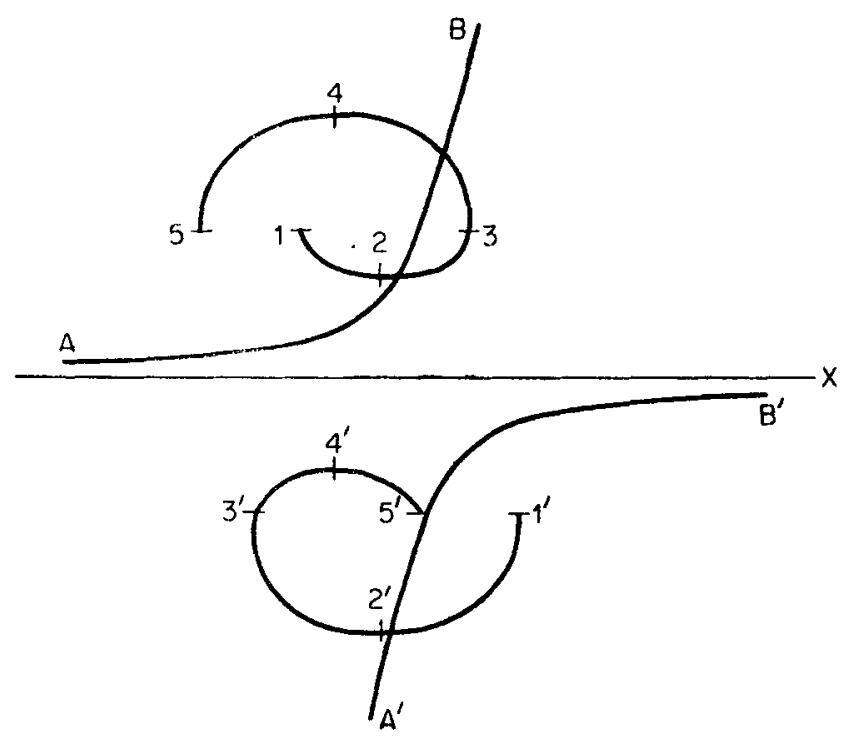

Figure 2. Generation of the nodoid curves $1-5$ and $1^{\prime}-5^{\prime}$ as the focal roulettes of a pair of hyperbolae $A B$ and $A^{\prime} B^{\prime}$ rolling on the line $X$.

have shifted one period to the left, although the mation of the contact point has always been towards the right.

The generation of nodoid surfaces from the hyperbolic focal roulette is best considered in two parts. Revolution of the sections 123 and $3^{\prime} 4^{\prime} 5^{\prime}$ about the rolling path generates an anticlastic surface in which the larger of the two principal curvatures (i.e., the smaller radius of curvature) lies on the side away from the axis. For the sections 345 and 1 ' 2 ' 3 ' both principal curvatures lie on the side nearer the axis. A fly walking from 1 to 5 would observe that the larger curvature was always on its right. We shall refer to the anticlastic and synclastic portions of the surface as nodoid $I$ and II respectively.

The significance of this is seen in relation to capillarity. A portion of the nodoid I might form a capillary surface bounding a drop of a wetting liquid surrounding a point of contact between two spheres (figure 3a). Using the Laplace equation with its ustal sign convention, the liquid is seen to be at reduccd pressure. (This fact is mainly responsible for the cohesion of moist powders.) The nodoid II, on the other hand, might be formed surrounding a drof of mercury pressed between parallel glass plates (figture $3 b$ ). The Laplace equation now indicates that such a drop will be subject to an increased pressure, tending to resist the approach of the solid surfaces. IIt may be noted that capillary forccs between solid bodies linked by a liquid bridge may conveniently be calculated with the aid of equation (11)].

Of the other four axisymmetric surfaces, three (sphere, cylinder, unduloid) have their largest curvature on the side of the axis (figure $3 c-e$ ); thus, when they enclose a liquid, the capillary pressure is positive. The catenoid has the singular property that its two curvatures are everywhere equal in magnitude and opposite 


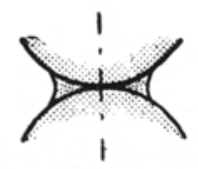

(a)

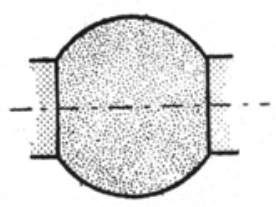

(c)

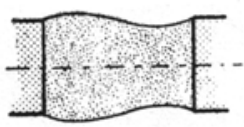

(e)

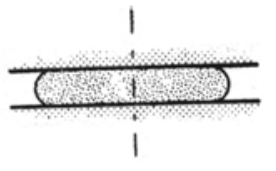

(b)

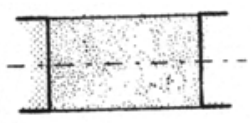

(d)

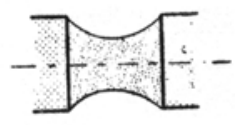

(f)

Figure 3. (a) Anticlastic nodoid $(\triangle P<0)$. (b) Synclastic nodoid $(\triangle P>0)$. (c) Sphere $(\triangle P>0)$. (d) Cylinder $(\triangle P>0)$. (e) Unduloid $(\triangle P>0)$. (f) Catenoid $(\triangle P=0)$.

in sign. It is thus a surface of zero mean curvature, and generatcs no capillary pressure (figure $3 \mathrm{f}$ ).

Further insight can be gained by considering the rolationships between the two principal curvatures, $k_{1}$ and $k_{2}$, for various classes of surface. Here $k_{1}$ is the curvature of the meridian curve, and $k_{2}$ is the curvature produced by rotation. The mean curvature is defined as

$$
C=k_{1}+k_{2}=1 / r_{1}+1 / r_{2},
$$

and, for such surfaces, is necessarily constant. In addition, we have

$$
\begin{array}{ll}
k_{1}=k_{2}>0 & \text { (sphere) } \\
k_{2}>k_{1}=0 & \text { (cylinder) } \\
0>k_{1}=-k_{2} & \text { (catenoid) }
\end{array}
$$

where a curvature is positive if its radius of curvature is dircetcd towards the axis of symmetry. Thus, in a plat of $k_{1}$ agsinst $k_{2}$ (figure 4) these surfaces are represented by straight lines, as indicated.

Taking equations (19), (20) and (21), we see that the sphcre and cylinder are singular surfaces ; for a given value of $C$ there is only one point on the appropriate line in figure 4 that will describe the surface. For these surfaces, not only is the sum $\left(k_{1}+k_{2}\right)$ everywhere constant, but so are $k_{1}$ and $k_{2}$ individually. The catenoid has an additional degrce of freedom: $k_{1}$ and $k_{2}$ can vary from point to paint, while satisfying (19) and (22), and so any catenaidal surface is represented not by a point but by a finite length of the appropriate line in figure 4 . 




Figure 4. Relationships between the two principal curvatures $k_{1}$ and $k_{2}$, for axisymmetric homoclastic surfaces.

The unduloid and nodoid possess yet another degree of freedom, in that not only do $k_{1}$ and $k_{2}$ vary over the surface, but also their sum $(=C)$ is nat restricted to a unique value, as it is for the catenoid $(C=0)$. Thus, these surfaces are represented by areas in figure 4 .

The anticlastic nodoid I always has $C$ negative:

$$
k_{1}<-k_{2}<0
$$

and so lies in the lowest actant of figure 4. The syrclastic nadoid II abcys

$$
k_{1}>k_{2}>0 \text {, }
$$

and so lies in the uppermost octant of figure 4.

The unduloid obeys

$$
\left|k_{1}\right|<k_{2}
$$

and lies in the remaining quadrant.

The other half of the field, not shown in figure 4, represents the family of surfaces with $k_{2}<0$, i.e., those in which the liquid phase lies ou tside the bounding surface. The appropriate diagram is obtained by rotating figure 4 through $180^{\circ}$ in its plane (ar by multiplying both axes by -1 ).

Any line of slope -1 drawn on figure 4, or its intersection with one of the lines representing a singular surface, i.e., sphere or cylinder, represents a solution to equation (19), and hence uniquely defines an equilibrium surface. However, it turns out that not all such surfaces are stable in the sense indicated by equation (12). They all represent extreme values of effective area, but some are local maxima. The stability conditions, when the surface is bounded in the axial direction by circular lines of fixed and equal diameter $D$, depend on the axial length $L$ and on the valume $V$ enclosed by the surface. Figure 5, adapted from 


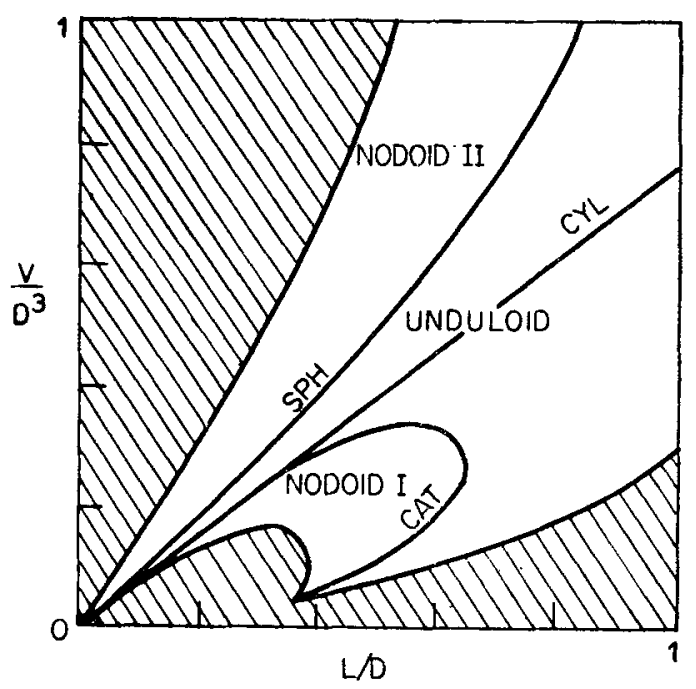

Figure 5. Portion of stability field of axisymmetric homoclastic surfaces. In the shaded regions, no stable equilibrium surfaces exist. The line representing the cylindrical surface meets the lower boundary of the unduloid region at $L / D=\pi$.

Da Riva and Martinez (1979), illustrates a portion of the stability ficld, together with regions for which no stable equilibrium homoclastic surface exists to contain a given valume. In general, a periadic surface like the undulojd bccomes unstable when its length exoceds one full wavelength. A surface containing portions of both types of nadoid, even though of uniform curvature, is similarly unstable. (The instability of a cylindrical surface whose length exceeds its circumference $L / D=\pi ; V / D^{3}=\pi^{2} / 4$ ) is well-known (Plateau 1873). Similarly, the catenoid is only stable when the tangents at its extremities intersect before reaching the axis of ratational symmetry (Maxwell 1876).

A fuller discussion of stability would take into account the fact that when the bo undary constraints are changed (e.g., constant contact angle rather than constant end diameter, or constant pressure rather than canstant volume), the stability limits also ohange. However, the oase discussed in detail is the one most closely related to various Spacelab experiments, to be considered in $\$ 3$.

\section{Relevance to Spacelab experiments}

In the early development of the Spacelab programme, heavy emphasis was laid on the possibility of commercially exploiting microgravity conditions in. the field of crystal growth. Although the emph,asis has since shifted, the present materials science programme still includes several studies of crystal growth by various techniques where removal of gravity may produce significant effects. It also includes a substantial programme of basic fluid physics experiments, several of which are aimed at improving fundamental understanding of the fluid mechanics of crystal growth processes, 


\subsection{Crystal growth}

Two of the techniques commonly used terrestrially for growing crystals from the melt are the floating zone and Czochralski methods. In the floating zone process, sometimes called zone melting or zone refining, a rod of raw material with a pure seed crystal at one end is progressively melted and re-solidified within a short heated zone, which is caused to travel slowly along the rod from the seeded end (figure 6). Impurities tend to be excluded at the solidification front and therefore are concentrated in the melt as the molten zone progresses along the rod.

Under normal gravity the dimensions of the molten zore - and rer.ce the size of the crystal that can be grown-are controlled by the need to keep the reciprocal Bond number small in order to preserve the stability of the zone. In microgravity the dimensions of the zone can be increased at will within the stability field of which figure 5 shows a small part: thus, much larger crystals can be grown.

However, the fluid-physical phenomena involved, even in microgravity, are complex and poorly understood. The axial gradients of temperature and composition will give rise to gradients of surface tersion, which will induce Marangoni convection within the zone. Moreover, the rod is usually rotated in order to reduce circumferential temperature-gradients. On earth, this may also exert a clinostatic action on a horizontal zone, hindering the accumulation of dense material in the bottom of the zone. However, this also means that the reciprocal of the rotational Bond number must be kept small enough so that the zone's stability is not impaired in microgravity.

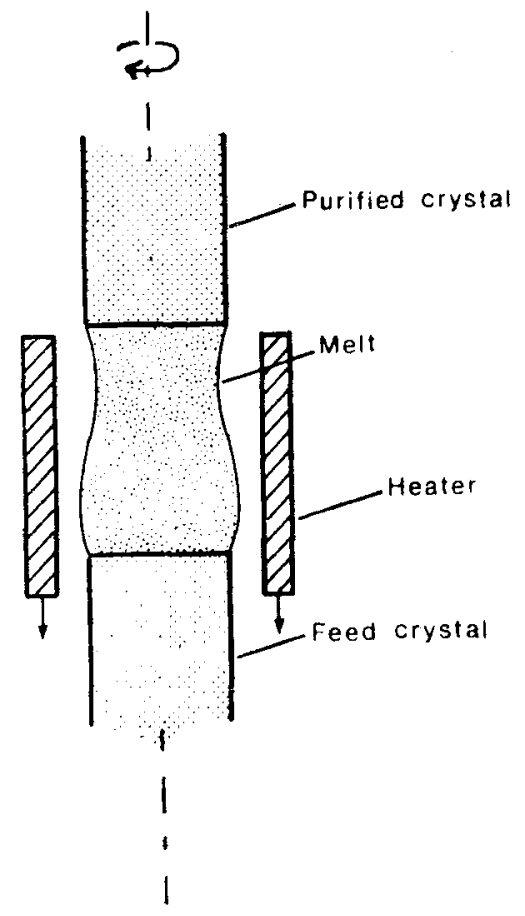

Figure 6. Floating zone method of crystal growth. 


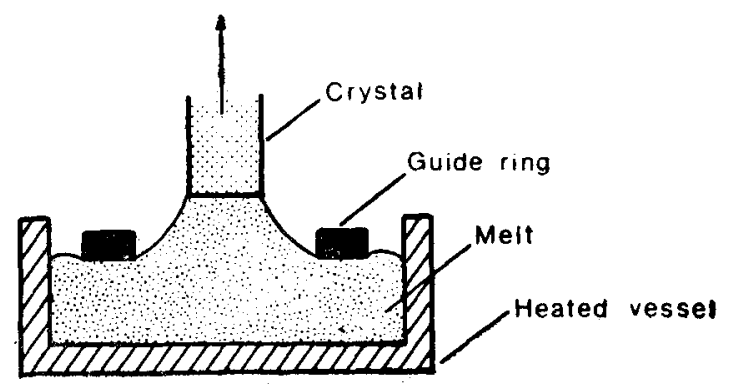

Figure 7. Czochralski method of crystal grawth.

In the Czochralski method (figure 7) a seed crystal is dipped into a melt to form a meniscus and is then slowly raised. Meanwhile, beat is extracted to cause solidification to take place at a stationary height above the free liquid surface.

On earth, gravity again limits the size of the melt meniscus and (with the orientation of figure 7) tends to produce unstable convection in conjunction with the imposed temperature-gradient.

Under microgravity the meniscus must adopt one of the axisymmetric configurations of $\$ 2.3$. It must be constrained to meet the seed crystal in a prescribed line and some appropriate constraint must also be applied in the region where it leaves the melt. In fact, there is no significant operational distinction hetween the floating zone and Czochralski methods when applied in microgravity. Once again, surface tension gradients in the meniscus may generate internal flows.

Results of earlier alloy solidification and crystal growth studies conducted in weightlessness have sometimes indicated improvements in quality, $\mathrm{k}$ ut they also show many puzzling features (McKannan 1977). The proposed Spacelab experiments recognise the need to work with simpler and tetter-characterised systems, under closely controlled conditions. At the same time, the planned series of experiments in fluid physics should improve fundamental understanding of the fluiddynamic phenomena involved in crystal growth.

\subsection{Fluid physics experiments}

Most of the fluid physics experiments to be carried in the first scientific payload of Spacelab will be conducted in the fluid physics module (FPM), described elsewhere in this volume. Of these, the investigations of Marangoni flows (principal investigator Professor L G Napolitano, University of Naples) and of the stat le configurations of rotating floating zones (principal investigator, Profess or I Da Riva, University of Madrid) are particularly relevant to crys tal growth phenomena.

In the first of these an axisymmetric model floating zone will be beld between parallel circular discs; the contact lines will be constrained to circles of fixed diameter by canthotaxis at a convex edge on the solid surface, with a chemical anti-spread barrier applied to the solid as an additional precaution. The working liquid, of about 1 litre volume, will be silicone oil: two oils of widely different viscosities but similar surface tensions will be used in successive runs. The ails will contain suspended glass microspheres as an aid to visualisation of internal flow patterns. 
By electrically heating one end-plate a temperature gradient will be established in the zone. Although buoyancy-driven convection will be practically non-existent in the microgravity of Spacelab, it is anticipatted that the thermally-induced surface tension gradient will excite significant Marangoni convecticn, eventually extending throughout the body of the fluid. Terrestrial experiments with zones of small dimensions have already demonstrated such flows, thou gh not, of course, in the absence of buoyancy forces (Chun and Wucst 1979). Unfortunately, the silicone oils, which were selected as warking fluids on the grounds of safety considerations (which are rigidly applied throughout Spacelab) have only small temperature coefficients of surface tensicn, and sc the Marangoni number that can be attained is rather low. Nevertheless, the experiment is expected to yield important information on the dynamics of the flow as it evolves towards the steady circulation accompanying a steady heat flux. Other experiments in the same series will investigate the possible flows resulting from imposition of a gradient of electric patential of the order of $10 \mathrm{~V} \mathrm{~cm}^{-1}$.

The second group of experiments will concern the stability of rotating liquid zones. Each experiment of the main series will consist of three parts:

3.2 (i) formation of a static zone of prescribed volume and configuration

3.2 (ii) gradual imposition of rotation. ("spin-up")

and 3.2 (iii) steady (solid-bady) rotation.

3.2a. During formation of the zone, the calculated predictions shown in figure 5 can be verified. When a zone has been brough,t to its static stability limit, the dynamics of rupture can be studied by high-speed photography, both of changes in external configuration and of internal flows, using the same tracer system as before.

3.2b. The spin-up problem has been of interest in many contexts for over half a century (see the review by Benton and Clark 1974). However, the particular situation encountered here, involving a deformable liquid surface anchored to a fixed line of contact, has resisted analysis except for the earliest stages of flow evolution (Da Riva and Martinez 1979). Ohservations, by both flow-tracing and profile photography, will therefore be of great interest.

3.2c. The effect of steady rotation of a floating zone is generally to reduce the domain of stability. An initially cylindrical zone of lengtr, $L$ and radius $R$ becomes unstable to axisymmetric disturbances when the rotation rate $\Omega$ reaches a critical value:

$$
\left(\Omega_{\mathrm{crit}}\right)^{2}=\left(\frac{\sigma}{\rho R^{3}}\right)\left(\frac{1-\lambda^{2}}{\lambda^{2}}\right)
$$

where $\quad \lambda=\frac{L}{2 \pi R} \leqslant 1$.

(Note that the classical limit $\lambda=1(\$ 2 \cdot 2)$ is recovered at $\left.\Omega_{\text {crit }}=0\right)$.

However, an improvised experiment conducted on board Skylab IV in 1974 by the astronaut E G Gibson (Carrtthers et al 1977) revealed that a rotating liquid zone can also become unstable to non-axisymmetric disturbances, going into a 
motion like that of a skipping rope, analogous to the whipping of an eccentrically mounted shaft. The two modes of instability have been termed "amphora" and " $C$-mode", with reference to their respective shapes.

Da Riva and Martinez (1979) have shown by a first-order linear analysis that the $C$-mode instability first appears at

$$
\left(\Omega_{\mathrm{crtt}}\right)^{2}=\left(\sigma / \rho R^{3}\right)\left(1 / 4 \lambda^{2}\right) .
$$

An analogous expression [equation (3) of Carruthers et al (1977), given without justification] reduces to

$$
\left(\Omega_{\text {crtt }}\right)^{2}=\left(\sigma / \rho R^{3}\right)\left(1 / 16 \lambda^{4}\right)
$$

but the error could be typographical.

Thus, the two stability boundaries cross at the value $\lambda=\sqrt{3 / 2}$ which satisfies equations (26) and (28). For $\sqrt{3 / 2}<\lambda<1$ the amphora instability is the first to be encountered by a cylindrical zone being slowly accelerated into axial rotation. For shorter zones higher rotation rates can be reached and the $\boldsymbol{C}$-mode instability is ercountered first.

The FPM experiments will provide a test of these predictions as well as information on rotating zones whose resting słape is not cylirdrical, for which no calculations have yet been performed.

\section{References}

Benton E R and Clark A 1974 Ann. Rev. Fluid Mech. 6257

Carruthers J R, Gibson E G, Klett M G and Facemire B R 1977 Prog. Astronaut. Aeronaut. 52 207

Cayias J L, Schechter R S and Wade W H 1975 Adsorption at interfaces (ed.) K L Mittal, ACS Symposium Series, Vol. 8, p. 234

Chun Ch H and Wuest W 1979 Materials Science in Space, Proc. 3rd European Sympasium ESA SP-142, p. 283

Da Riva 1 and Martinez I 1979 Materials Science in Space, Proc. 3rd European Symposium, ESA SP-142, p. 67

Defay R, Prigogine I, Bellemans A and Everett D H 1966 Surface tension and adsorption (London: Longmans Green)

Everett D H and Haynes J M 1972a Z. phys. Chem. 8236

Everett D H and Haynos J M 1972b J. Colloid Interface Sci. 38125

Gibbs J W 1876-1878 Trans. Connecticut Acad. 108 343. These papers are collected in The Scientific Papers of $J$ Willard Gibbs, Vol. 1, 1906 pp. 55-353 (London: Longmans Green) and reprinted by Dover Publications in 1961

Guggenheim E A 1957 Thermodynamics 3 edn. (Amsterdam: North Holland) p. 46

Haynes J M 1977 Prog. Astronaut. Aeronaut. 5257

Johnson R E 1959 J. Phys. Chem. 631655

Maxwell J C 1986 Capillary Action, in Encyclopaedia Britannica 9th edn. Vol. 5 p. 56

McKannan E 1977 Progr. Astronaut. Aeronaut. 77383

Ono S and Kondo S 1960 Encyclopaedia of Physics (ed.) Flügge (Berlin : Springer) 10134

Plateau J A F 1873 Statique expérimentale et théorique des liquides soumis aux seules forces moleculaires (Paris: Gauthier Villars)

Vonnegut B 1942 Rev. Sci, Instrum. 136 